\title{
Planning for shortages? Net Present Value Analysis for a Deteriorating Item with Partial Backlogging
}

\author{
Yousef Ghiami ${ }^{1}$ \\ Faculty of Economics and Business Administration, \\ VU University Amsterdam, 1081 HV, The Netherlands, Y.Ghiami@vu.nl \\ Patrick Beullens \\ Mathematical Sciences and Southampton Business School and CORMSIS, \\ University of Southampton, SO171BJ, United Kingdom, P.Beullens@soton.ac.uk
}

\begin{abstract}
This paper develops inventory models to help answer strategic questions concerning whether planning for shortages offers financial benefits. A production-inventory system producing a deteriorating product in batches at a finite production rate with partial backordering is considered. Customers pay a deposit when placing a backorder. Backordered items receive a discount on the sales price. As lost sales may lead to customers not returning, the demand rate may depend on the fraction of lost sales. We develop a cash-flow based profit maximising Net Present Value (NPV) model without the inventory cost parameters commonly used in this context: unit holding cost, unit backorder cost, unit deterioration cost, and unit lost sales cost. The model finds the optimal inventory policy just like NPV models that discount the traditional parameters but has the advantage of not needing to estimate the value of the traditional parameters. It is shown that in models based on discounting the traditional parameters, the parameters are not exogenously determinable but are non-trivial functions of non-financial endogenous system parameters such as the production rate, annual demand rate, and backorder rate. Extensive numerical experiments illustrate how cash-flow NPV models provide insights into the value of planning for shortages and strategic choices about the design of the production-inventory system. It also provides insight into the classical problem of how to interpret unit backorder cost and unit lost sales cost. The study indicates that these insights cannot be reliably obtained from NPV models based on discounting unit backorder costs and unit lost sales costs.
\end{abstract}

keywords: Net Present Value; deteriorating inventory; finite production rate; shortages

\footnotetext{
${ }^{1}$ Corresponding Author

This manuscript has been accepted on April $26^{\text {th }} 2016$ for publication in the International Journal of Production Economics subject to standard editing, DOI: 10.1016/j.ijpe.2016.04.021
} 


\section{Introduction}

This paper looks into the question of how deterministic inventory models can provide quantitative insights into the financial merit of system design choices, in particular whether a firm can derive financial benefits from planning for shortages. In the process the paper also addresses a long outstanding question in the literature about how to interpret the classic inventory parameters unit backorder cost and unit lost sales cost.

Should a firm plan its inventory replenishments such that shortages purposefully occur? Intuition says that since shortages affect revenues it might make sense to minimise their occurrence. On the other hand, backorders reduce inventory holding costs and therefore the answer relies on a trade-off. In order to capture this trade-off, inventory models are to allow for the possibility of shortages. To establish the financial value of planning for shortages, the model should price the cost of shortages with some degree of accuracy. This issue has not received much attention in the literature despite being of crucial importance for applying models to practice.

Deterministic inventory models from the literature which allow for shortages to occur indicate that it may be worthwhile to plan for backorders even if some of the demand during a period of shortages is lost, see e.g. Pentico and Drake (2009). The value of such predictions, however, relies on the accuracy of the numerical values of the model parameters, in particular on the values used for the unit backorder cost $h_{b}$ and unit lost sales cost $h_{l}$. It further depends on the definition of these parameters, in particular whether $h_{b}$ is taken to be a cost per unit of product and unit of time or a cost per unit of product ${ }^{1}$. While it is recognised in the literature that 'quantifying these parameters in practise is difficult' (Winston, 1994), the inventory literature does not address the question of what these parameters actually represent, i.e., how to set their values accurately in some particular application. The findings in this paper support the view that without additional guidelines, these models remain somewhat inadequate for finding an optimal inventory

\footnotetext{
${ }^{1}$ For example, if in the classic Economic Order Quantity model $h_{b}$ is defined per unit of product, then depending on its value the optimal strategy is either not to have stockouts or not to perform the activity at all. This requires an estimate of whether $h_{b}$ is above or below a threshold value determined by classic EOQ model parameters. When adopting the more widely used definition that $h_{b}$ is a cost per unit of product and unit of time, an optimal policy always plans for backorders if there are no lost sales, and to know the optimal backorder level requires having an accurate estimate of $h_{b}$. See e.g. Zipkin (2000).
} 
policy for a particular firm or for deriving insights into the potential financial benefits of shortages or other system design choices.

This paper contributes to the literature by demonstrating the value of inventory models based on cash-flow functions. Cash-flow based models do not make use of the difficult to determine traditional inventory cost parameters such as $h_{b}$ and $h_{l}$. Instead, revenue and cost cash-flows expressed as functions of the logistics design and the payment structures that a firm adopts with external parties form part of an objective function that maximises the Net Present Value (NPV) of this activity. We demonstrate that such models are more suited to determine the financial worth of strategic design choices of an inventory system, and that from a comparison with NPV models using the traditional parameters instead insight into the nature of these traditional parameters can be obtained. Parameters in a model, in the strict sense, should be exogenously determinable. In the system examined in this paper, we find that the traditional parameters $h_{b}$ and $h_{l}$ would be non-trivial functions of other endogenous system parameters. In contrast, all parameters in the cash-flow based NPV model developed are still exogenously determinable.

We consider a production-inventory system where a deteriorating product is produced in batches at a finite production rate and where a stock-out can result in either a backorder or a lost sale. This case is interesting since intuition tells us that these features will in general affect the value of planning for shortages and in particular that if a product deteriorates the incentive to disinvest in inventory and use the mechanism of backorders will increase.

The management of an inventory of deteriorating items has been widely addressed in the literature. Surveys of deterministic and stochastic models include Nahmias (1982), Raafat (1991), Goyal and Giri (2001), Li et al. (2010) and Bakker et al. (2012). Papers studying deterioration with partial backlogging with an NPV objective function can be partitioned into profit maximising versus cost minimising models. The first class includes Wee and Law (2001); Hou (2006); Dye et al. (2007); Singh et al. (2009); Yang et al. (2010); Hsieh and Dye (2010); Hou and Lin (2011) and Yu (2013). As demand is either priceor stock-dependent, it is natural to consider revenue streams explicitly and adopt the objective of profit maximisation. Cost minimising NPV models include Jaggi and Aggarwal (1994); Aggarwal and Jaggi (1995); Liao et al. (2000); Sarker et al. (2000); Chung and Liao (2006); Chang et al. (2010); Liao and 
Huang (2010); Balkhi (2011) and Taleizadeh and Nematollahi (2014). Single warehouse situations are considered, with or without an element of credit or permissible delays in payment. Other papers deal with a two-warehouse setting, as in Yang (2004, 2006, 2012); Wee et al. (2005); Hsieh et al. (2008) and Singha et al. (2013). A small minority of work concerns inventory management across multiple echelons in the supply chain, see e.g. Law and Wee (2006) and Lo et al. (2007). Provided that the potential changes on revenues are considered, e.g. that a revenue loss is accounted for as a cost, the objective of cost minimisation will be equivalent to profit maximisation.

In a context of item deterioration, backorders and lost sales, classic inventory theory principles indicate that next to the unit holding cost $h$, other relevant inventory parameters are the unit deterioration cost $h_{d}$, the unit backorder cost $h_{b}$ and the unit lost sales cost $h_{l}$. We indeed find these four classic parameters in the above literature, where their impact on the NPV objective function is found from discounting these costs over relevant inventory functions and time periods. A distinction can be made between models that include the opportunity cost of holding stock in $h$, as in e.g. Yang (2004, 2006, 2012), and models that do not include the opportunity cost in $h$, as in e.g. Moon et al. (2005); Jaggi et al. (2006); Law and Wee (2006); Lo et al. (2007); Hsieh et al. (2008); Taleizadeh and Nematollahi (2014) and Chern et al. (2008). In the latter case, the effect of the unit production cost is explicitly included in the formulation. (These approaches are further illustrated in Section 4.1 and Section 4.2, respectively.)

In contrast to the existing literature in this field, the main model in this paper derives its NPV objective function from cash-flow functions, excluding the use of the classic parameters $h, h_{d}, h_{b}$ and $h_{l}$. This approach requires modelling what triggers costs and revenues, and their timing, more accurately. In particular, we consider the situation in which customers may pay a deposit for backordered items or may receive a reduction on the sales price as a compensation for having incurred the backorder. Based on our experience, we can find these elements as part of backorder policies in real situations, including in the hand-made production and sales of chocolates by some artisanal Belgian chocolatiers where customers pay deposits for their backorder in order to secure the order, and in the situation of firms in military supply chains who are subject to financial compensation rules for backorders in their contract with the armies they supply. 
The main draw-back of using classic inventory cost parameters is that it does not produce insight into what these parameters actually represent and how to find good numerical values for them. The first clear evidence of this is given in the article of Grubbström (1980), which together with Grubbström (1967) also laid the foundations of a cash-flow based Net Present Value (NPV) approach to studying production-inventory systems. Grubbström (1980) shows how this technique leads to a specification of $h$ as a function of financial system parameters, and that in some systems this functional relationship deviates significantly from the convention that $h$ is to be based on money invested into stock. An extension of this approach is made in Beullens and Janssens (2011), who introduce the Anchor Point in a model and show that its placement in a supply chain may affect the specification of $h$. In Beullens and Janssens (2014), the technique of using these NPV models as a reference to gain insight into classic inventory models is formalised as NPV Equivalence Analysis (NPVEA). Its application has led to refinements to classic inventory theory when applied to certain contexts, see Boyaci and Gallego (2002); Teunter and van der Laan (2002); and Beullens (2014). To our knowledge, NPVEA has not been applied to find interpretations of models that use the four parameters $h, h_{b}, h_{l}$ and $h_{d}$. In fact, no NPV interpretations are available for any of these parameters but $h$, and only one has been identified for $h_{b}$, see Grubbström (1998), in a system without item deterioration. In the context of the NPVEA literature, this paper contributes by showing that $h_{b}$ and $h_{l}$ in the system studied in this paper are also functions of non-financial system parameters.

The application of NPVEA in this paper shows that if customers would be financially compensated for incurring a backorder, $h_{b}$ and $h_{l}$ not only depend on financial parameters but also on non-financial system parameters such as the production rate, the demand rate and the probability of whether a shortage would produce a backorder or a lost sale. We also find that the unit lost sales cost $h_{l}$ is a function of the financial compensation given to customers with backorders. These findings motivate us to develop and promote cash-flow based NPV models as a viable alternative to models based on discounting classic inventory parameters.

The paper is further organised as follows. Section 2 introduces the system under study, and Section 3 develops the corresponding mathematical equations specifying the cash-flow based NPV reference model. In Section 4 we derive two NPV models based on discounting the classic inventory parameters. It is shown 
that equivalence of these modelling approaches with the NPV reference model holds, subject to a set of conditions from which a useful specification of the classic cost parameters follows. Numerical examples in Section 5 illustrate how the cash-flow NPV model can be used to gain insight into the value of planning for shortages, and into the impact of other system design choices. The equivalence theorems obtained indicate that such insights cannot be reliably obtained from an NPV model based on the classic parameters when their functional relationships with other system parameters are not known. Conclusions are presented in Section 6.

\section{Description of the system}

Notation:

- $p$, sales price per unit of product;

- $y$, demand rate per unit of time when product is in stock;

- $\beta$, fraction of demand during stock-outs resulting in backorders $(0 \leq \beta \leq 1)$, the remaining part are lost sales;

- $s$, set-up cost to initiate a production run;

- $c$, cost to produce a unit of product;

- $R$, finite production rate of production process per unit of time $(R>y)$;

- $T_{i}$, component of inventory cycle time $T, i=\{1,2,3,4\}$ (decision variables);

- $T$, inventory cycle time, $T=T_{1}+T_{2}+T_{3}+T_{4}$;

- $\theta$ and $\gamma$, scale and shape parameters of Weibull deterioration rate distribution;

- $d$, unit cost to dispose of a deteriorated product (could also be negative, i.e. a unit salvage value);

- $g$, deposit paid by customers to secure backordered product $(g \geq 0)$; 
- $r$, compensation paid to customers for backordered product $(r \geq 0)$;

- $f$, out-of-pocket warehouse costs per unit of product and unit of time $(f \geq 0)$;

- $b$, out-of-pocket cost per unit of outstanding demand backordered and unit of time $(b \geq 0)$;

- $\pi$, out-of-pocket cost per unit of demand lost $(\pi \geq 0)$;

- $\alpha$, opportunity cost of capital rate.

\subsection{The activity}

When the product is in stock, the demand rate $^{2}$ is $y$. Whenever the item is not in stock, the demand rate drops to $\beta y$; this fraction of demand is met with backorders. The remaining fraction $(1-\beta) y$ are lost sales.

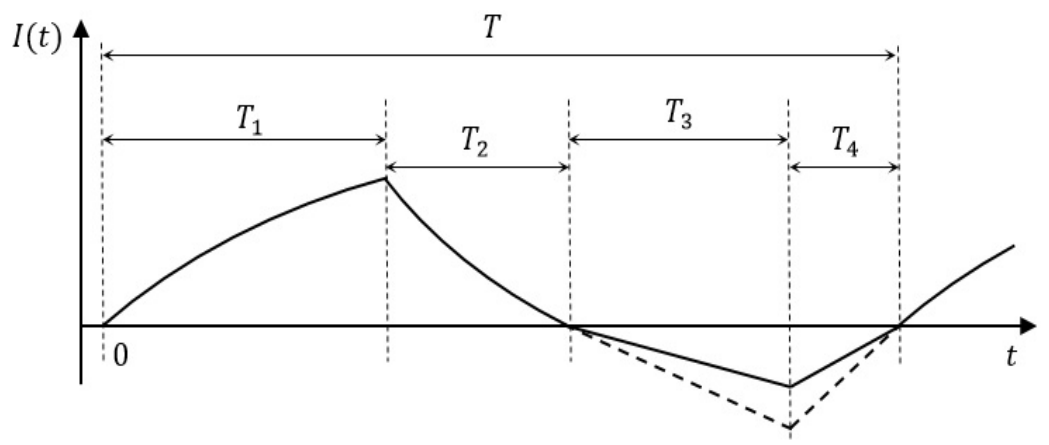

Figure 1: Inventory level of the system

Figure 1 displays the stock position of serviceable products as a function of time. The system starts at time $t=0$ with zero inventory and with the initiation of the first production run which generates products at rate $R$ for a time $T_{1}$, during which all demand is met and since $R>y$ stock $I(t)>0$ is built up. At time $t=T_{1}$ the production stops. During the period $T_{2}$ demand is met from stock.

The inventory level $I(t)$ in period $T_{1}$ increases and in $T_{2}$ decreases in a non-linear fashion due to an increased number of items in stock deteriorating. This process is modelled, in a deterministic fashion, as follows. Items that have deteriorated are immediately removed from serviceable inventory, and demand

\footnotetext{
${ }^{2}$ The model is easily extended to consider this rate to be a function of sales price $y(p)$
} 
from serviceable stock is met in a First-Come-First-Serve (FCFS) manner. Given a non-negative inventory level $I(t)$, where $t$ measures the time from the production run initiation, the rate of deterioration is $\theta \gamma t^{\gamma-1}$, where $\theta$ and $\gamma$ are two suitably chosen constants. This corresponds to the failure rate of a Weibull distribution $f(t)=\theta \gamma t^{\gamma-1} e^{-\theta t^{\gamma}}$, where $\theta$ and $\gamma$ are the scale and the shape parameter, respectively. In case that $\gamma=1$, the failure rate is constant and characterises a negative exponentially distributed deterioration process.

At $t=T_{1}+T_{2}$ stock has reduced to zero and the demand rate drops to $\beta y$, and this demand is backlogged. A shortage level $B(t)=-I(t)>0$ is built up according to the rate $\beta y$ for a length of time $T_{3}$. Note that the dashed line in the time segment $T_{3}$ in Figure 1 indicates the level of backorders when there would be no lost sales, i.e. in the case that beta $=1$. At time $t=T_{1}+T_{2}+T_{3}$ the production process is re-initiated. During $T_{4}$ the demand that arises at rate $\beta y$ is instantaneously satisfied from this production process, while the excess production capacity at rate $(R-\beta y)$ goes towards satisfying the backlogged demand in a FCFS manner and hence at this rate the shortage level $B(t)$ is being reduced. At $t=T_{1}+T_{2}+T_{3}+T_{4} \equiv T$ the inventory position is back at zero, at which point all demand that occurred in the past has been satisfied. The logistics process is back in a state it was in at the beginning $t=0$ safe the fact that production has already started. This process repeats itself at infinitum.

\subsection{The cash-flows}

The objective is to maximise the Net Present Value of relevant future cash-flow functions associated with the activity described in Section 2.1. For this purpose we examine how the firm exchanges cash-flows with the outside world. These are assumed to be as follows; see also Figure 2.

For any demand that arises when the stock position is strictly positive, customers pay the unit price $p$ instantaneously. This produces an income at the annuity stream level $p y$ during the periods associated with $T_{1}$ and $T_{2}$. Payment is also immediate during the period associated with $T_{4}$ for the demand generated at rate $\beta y$ during that period, providing an income at the annuity stream level $p \beta y$. During the period of stock-out associated with $T_{3}$, demand is partially backlogged. Customers may pay a deposit $g \geq 0$ the moment they raise the demand. In addition they may be promised a reduction $r \geq 0$ on the sales price 


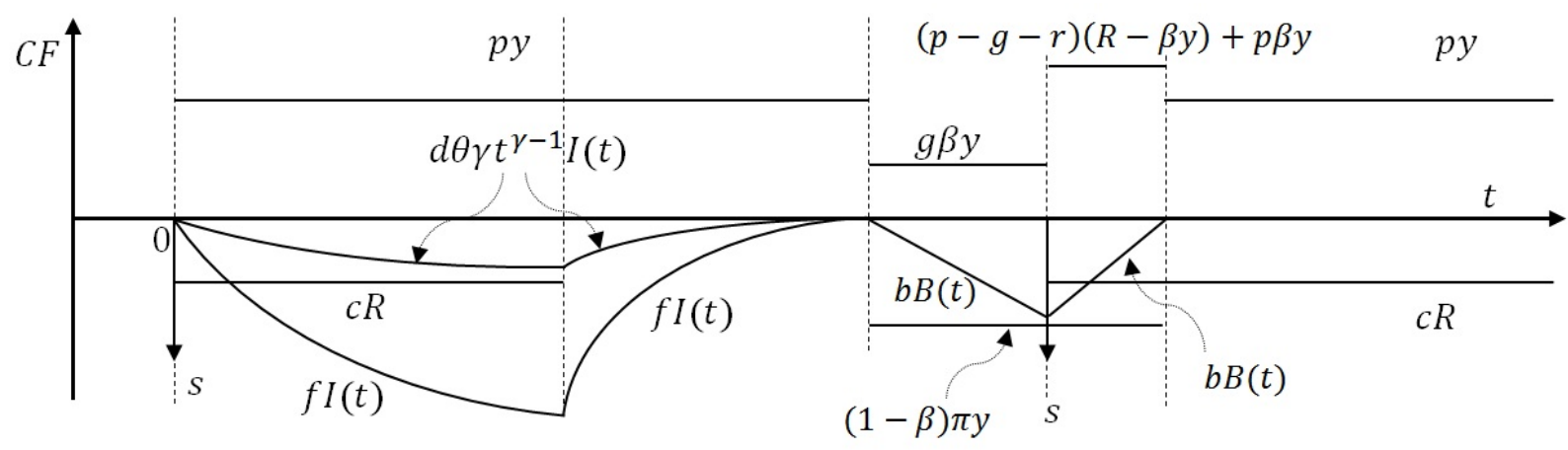

Figure 2: Cash-flows of the profit maximisation model

as a compensation for the waiting. A customer for which a backorder of a product was created will thus pay $p-g-r$ when receiving the product. In $T_{4}$ the backlog is reduced at the rate $(R-\beta y)$ and the corresponding annuity stream level income is $(p-g-r)(R-\beta y)$.

The firm incurs the set-up cost $s$ the moment production initiates. As production occurs at rate $R$, the firm's variable unit production cost $c$ is incurred at the annuity stream level $c R$ for a time spanning the period $T_{1}$ in the first production run, and for a time spanning $T_{4}+T_{1}$ for any subsequent production run. During the period spanning $T_{1}+T_{2}$ the items that deteriorate at any time $t$ are immediately removed from stock and disposed of for a cost of $d$ per unit of product, immediately paid out to e.g. a recycling company.

During $T_{1}+T_{2}$ the firm incurs an out-of-pocket warehouse cost $f \geq 0$ per unit of product and unit of time, paid out instantaneously. During $T_{3}+T_{4}$ an out-of-pocket cost $\pi \geq 0$ per unit of demand lost is incurred as a continuous outflow of cash at the annuity stream level $(1-\beta) \pi y$. During $T_{3}$ and $T_{4}$ an out-of-pocket backorder cost $b \geq 0$ per unit of outstanding demand and unit of time is instantaneously incurred.

\section{Mathematical model of the system}

\subsection{Inventory and shortage levels}

At the start of the inventory cycle, production accumulates inventory while deterioration and demand decrease the inventory level. This pattern starts at time zero (for the first period) until the production 
stops at time $T_{1}$. The following differential equation represents the inventory level in this period:

$$
\frac{d I(t)}{d t}=R-y-\theta \gamma t^{\gamma-1} I(t), \quad 0 \leq t \leq T_{1}, I(0)=0 .
$$

By solving the differential equation (1), the inventory level in this period is obtained:

$$
I(t)=(R-y) e^{-\theta t^{\gamma}} \int_{0}^{t} e^{\theta u^{\gamma}} d u, \quad 0 \leq t \leq T_{1} .
$$

At time $T_{1}$ the production stops and the demand is covered using the produced items; this pattern continues until the inventory level reaches zero at $T_{1}+T_{2}$ :

$$
\frac{d I(t)}{d t}=-y-\theta \gamma t^{\gamma-1} I(t), \quad T_{1} \leq t \leq T_{1}+T_{2}, I\left(T_{1}+T_{2}\right)=0 .
$$

The inventory level in this interval is hence as follows:

$$
I(t)=y e^{-\theta t^{\gamma}}\left(\int_{T_{1}}^{T_{1}+T_{2}} e^{\theta u^{\gamma}} d u-\int_{T_{1}}^{t} e^{\theta u^{\gamma}} d u\right), \quad T_{1} \leq t \leq T_{1}+T_{2} .
$$

As $I(t)$ takes on a unique value at $t=T_{1}$, there hence holds a relation between $T_{1}$ and $T_{2}$ obtained from (2) and (4); we further consider $T_{1}$ as a function of $T_{2}$, or $T_{1}=g\left(T_{2}\right)$, as implicitly given by this condition. (For an approximate solution, see Appendix A.)

During the out-of-stock period and before the production starts again, the shortage level increases as a percentage of the demand is backordered:

$$
\frac{d B(t)}{d t}=\beta y, \quad T_{1}+T_{2} \leq t \leq T-T_{4}, B\left(T_{1}+T_{2}\right)=0 .
$$

The shortage level within this period is therefore:

$$
B(t)=\beta y\left(t-T_{1}-T_{2}\right), \quad T_{1}+T_{2} \leq t \leq T-T_{4} .
$$


When the production starts at $T-T_{4}$ as the inventory level is zero a percentage of the demand $(1-\beta)$ is lost and the rest is met with no delay. The excess production capacity is used to satisfy the backorders accumulated between $T_{1}+T_{2}$ and $T-T_{4}$ and decreases the shortage level:

$$
\frac{d B(t)}{d t}=-(R-\beta y), \quad T-T_{4} \leq t \leq T, B(T)=0 .
$$

The corresponding shortage level is:

$$
B(t)=(R-\beta y)(T-t), \quad T-T_{4} \leq t \leq T .
$$

Considering the continuity at $t=T-T_{4}$, or that (6) and (8) should take the same unique value at this point, the following equation is obtained:

$$
T_{4}=\frac{\beta y}{R-\beta y} T_{3},
$$

therefore

$$
T=g\left(T_{2}\right)+T_{2}+\frac{R}{R-\beta y} T_{3} .
$$

In conclusion, we can take $T_{2}$ and $T_{3}$ as the independent decision variables for the firm since $T_{1}, T_{4}$ and $T$ then follow from above relationships. In particular: $T_{1}=g\left(T_{2}\right)$ is obtained from equating the right-hand sides of (2) and (4) for $t=T_{1} ; T_{4}$ from using (9); and $T$ from (10).

\subsection{Annuity streams of cash-flows}

As shown in Figure 2, a revenue at the rate of $p y$ is continuously received between 0 and $T_{1}+T_{2}$. The present value of this revenue for the first inventory cycle only, is given by:

$$
\begin{aligned}
R_{1} & =p y \int_{0}^{T_{1}+T_{2}} e^{-\alpha t} d t \\
& =\frac{p y}{\alpha}\left(1-e^{-\alpha\left(T_{1}+T_{2}\right)}\right) .
\end{aligned}
$$


The equivalent annuity stream of all such revenues received over an infinite of future inventory cycles, is:

$$
\begin{aligned}
A S R_{1} & =R_{1} \sum_{i=0}^{\infty} \alpha e^{-i \alpha T} \\
& =p y \frac{1-e^{-\alpha\left(T_{1}+T_{2}\right)}}{1-e^{-\alpha T}} .
\end{aligned}
$$

During the interval $T_{1}+T_{2} \leq t \leq T-T_{4}$, due to the out-of-stock situation, a percentage of the demand is backlogged and the customers pay a deposit $g$ to receive their item later when the production starts again. The corresponding annuity stream of this revenue is hence:

$$
\begin{aligned}
A S R_{2} & =g \beta y \int_{T_{1}+T_{2}}^{T-T_{4}} e^{-\alpha t} d t \sum_{i=0}^{\infty} \alpha e^{-i \alpha T} \\
& =g \beta y\left[\frac{1-e^{-\alpha\left(T-T_{4}\right)}}{1-e^{-\alpha T}}-\frac{1-e^{-\alpha\left(T_{1}+T_{2}\right)}}{1-e^{-\alpha T}}\right] .
\end{aligned}
$$

Revenues are also received in the first inventory cycle when production restarts at $T-T_{4}$, and until $T$. The first part of this revenue is to be associated with the delayed fulfilment of backlogged demand that arose in the interval $T_{1}+T_{2} \leq t \leq T-T_{4}$, and as this is sold at a discount, it generates revenue at the rate $(p-g-r)(R-\beta y)$; the second part with the immediate fulfilment of demand that arises in $T-T_{4} \leq t \leq T$, and thus produces revenue at rate $p \beta y$. The annuity stream of this revenue is given by:

$$
\begin{aligned}
A S R_{3} & =((p-g-r)(R-\beta y)+p \beta y) e^{-\alpha\left(T-T_{4}\right)} \int_{0}^{T_{4}} e^{-\alpha t} d t \sum_{i=0}^{\infty} \alpha e^{-i \alpha T} \\
& =((p-g-r)(R-\beta y)+p \beta y)\left[1-\frac{1-e^{-\alpha\left(T-T_{4}\right)}}{1-e^{-\alpha T}}\right] .
\end{aligned}
$$

The set-up cost of production $s$ is incurred at $t=0$ for the first production run, and then at a time $T_{4}$ earlier relative to the start of every subsequent inventory cycle. The relevant annuity stream is as follows:

$$
\begin{aligned}
S C & =\alpha s\left(1+e^{-\alpha\left(T-T_{4}\right)}+e^{-\alpha\left(T-T_{4}\right)} e^{-\alpha T}+e^{-\alpha\left(T-T_{4}\right)} e^{-2 \alpha T}+\ldots\right) \\
& =\alpha s\left(1+\frac{e^{-\alpha\left(T-T_{4}\right)}}{1-e^{-\alpha T}}\right) .
\end{aligned}
$$


The cost of production at rate $c R$ is incurred between $n T-T_{4}$ and $n T+T_{1}(n=1,2,3, \ldots)$ except for the first period in which the production takes place between 0 and $T_{1}$. The annuity stream of the production cost is:

$$
\begin{aligned}
P C & =\alpha c R \int_{0}^{T_{1}} e^{-\alpha t} d t+\alpha c R e^{-\alpha\left(T-T_{4}\right)}\left(1+e^{-\alpha T}+e^{-2 \alpha T}+\ldots\right) \int_{0}^{T_{1}+T_{4}} e^{-\alpha t} d t \\
& =c R\left[1+\frac{1-e^{-\alpha T_{1}}}{1-e^{-\alpha T}}-\frac{1-e^{-\alpha\left(T-T_{4}\right)}}{1-e^{-\alpha T}}\right] .
\end{aligned}
$$

The cost of warehouse space in the first period consists of two parts and considering the inventory level presented in (2) and (4), the corresponding present value of these costs are (for approximate solutions, see Appendix A):

$$
H C_{1}=f \int_{0}^{T_{1}} I(t) e^{-\alpha t} d t
$$

and

$$
H C_{2}=f \int_{T_{1}}^{T_{1}+T_{2}} I(t) e^{-\alpha t} d t .
$$

The warehouse cost over all future periods gives the annuity stream:

$$
H C=\frac{\alpha}{1-e^{-\alpha T}}\left(H C_{1}+H C_{2}\right) .
$$

Outstanding (unsatisfied) demand arises between $T_{1}+T_{2}$ and $T$, during which a backorder penalty cost $b$ per unit of item and time in incurred. Using the shortage levels presented in (6) and (8), the present value of this cost in the first inventory period is:

$$
\begin{aligned}
B C_{1} & =b \beta y \int_{T_{1}+T_{2}}^{T-T_{4}}\left(t-T_{1}-T_{2}\right) e^{-\alpha t} d t \\
& =\frac{b \beta y}{\alpha} e^{-\alpha\left(T_{1}+T_{2}\right)}\left[\frac{1}{\alpha}\left(1-e^{-\alpha T_{3}}\right)-T_{3} e^{-\alpha T_{3}}\right]
\end{aligned}
$$


and

$$
\begin{aligned}
B C_{2} & =b(R-\beta y) \int_{T-T_{4}}^{T}(T-t) e^{-\alpha t} d t \\
& =\frac{b(R-\beta y)}{\alpha} e^{-\alpha\left(T-T_{4}\right)}\left[T_{4}-\frac{1}{\alpha}\left(1-e^{-\alpha T_{4}}\right)\right] .
\end{aligned}
$$

Thus the annuity stream of this cost over an infinite horizon is:

$$
\begin{aligned}
B C & =\frac{\alpha}{1-e^{-\alpha T}}\left(B C_{1}+B C_{2}\right) \\
& =\frac{b R}{\alpha}\left[\frac{1-e^{-\alpha\left(T-T_{4}\right)}}{1-e^{-\alpha T}}\right]-\frac{b \beta y}{\alpha}\left[\frac{1-e^{-\alpha\left(T_{1}+T_{2}\right)}}{1-e^{-\alpha T}}\right]-\frac{b}{\alpha}(R-\beta y) .
\end{aligned}
$$

A penalty of $\pi$ per unit of lost sale is also due with immediate effect. The annuity stream of this out-of-pocket lost sale cost is:

$$
\begin{aligned}
L C & =\frac{\alpha}{1-e^{-\alpha T}} \pi y(1-\beta) \int_{T_{1}+T_{2}}^{T} e^{-\alpha t} d t \\
& =\pi y(1-\beta)\left[1-\frac{1-e^{-\alpha\left(T_{1}+T_{2}\right)}}{1-e^{-\alpha T}}\right] .
\end{aligned}
$$

A deteriorated item incurs a net cost $d \geq 0$ for the firm to cover the disposal or recycling fees, or represents a net revenue $d<0$ if the deteriorated item retains a salvage value that other industries are willing to pay. The annuity cost for disposal is given by:

$$
D C=\frac{\alpha}{1-e^{-\alpha T}} d \int_{0}^{T_{1}+T_{2}} \theta \gamma t^{\gamma-1} I(t) e^{-\alpha t} d t .
$$

Hence, the firm's annuity stream profit function to be maximised is:

$$
A S P=A S R_{1}+A S R_{2}+A S R_{3}-(S C+P C+H C+B C+L C+D C),
$$

where $T_{2}, T_{3}$, and $p$ are the firm's decision variables. 


\subsection{Special case: $\gamma=1$}

This section presents the special case that $\gamma=1$ (negative exponential deterioration) for which explicit analytical solutions, to be used in Section 4, can be obtained. The shortage level equations (6) and (8) and boundary condition (9) remain unaltered. Now the inventory levels as previously given by (2) and (4) are:

$$
I(t)=\frac{(R-y)}{\theta}\left(1-e^{-\theta t}\right), \quad 0 \leq t \leq T_{1}
$$

and

$$
I(t)=\frac{y}{\theta}\left(e^{\theta\left(T_{1}+T_{2}-t\right)}-1\right), \quad T_{1} \leq t \leq T_{1}+T_{2} .
$$

Continuity at $T_{1}$ for (26) and (27) results in the following boundary condition:

$$
e^{\theta T_{2}}=\frac{R}{y}-\left(\frac{R}{y}-1\right) e^{-\theta T_{1}} .
$$

The deterioration cost, previously (24), is now:

$$
\begin{aligned}
D C= & \frac{\alpha}{1-e^{-\alpha T}} d \theta \int_{0}^{T_{1}+T_{2}} I(t) e^{-\alpha t} d t \\
= & \frac{d(R-y)}{1-e^{-\alpha T}}\left[1-e^{-\alpha T_{1}}-\frac{\alpha}{\alpha+\theta}\left(1-e^{-(\alpha+\theta) T_{1}}\right)\right] \\
& +\frac{d y}{1-e^{-\alpha T}}\left[\frac{\alpha}{\alpha+\theta} e^{-\alpha T_{1}}\left(e^{\theta T_{2}}-e^{-\alpha T_{2}}\right)-e^{-\alpha T_{1}}+e^{-\alpha\left(T_{1}+T_{2}\right)}\right] \\
= & \frac{d \theta}{\alpha+\theta}\left[R \frac{1-e^{-\alpha T_{1}}}{1-e^{-\alpha T}}-y \frac{1-e^{-\alpha\left(T_{1}+T_{2}\right)}}{1-e^{-\alpha T}}\right] .
\end{aligned}
$$

The annuity stream of the holding cost can be found in a similar way:

$$
H C=\frac{f}{\alpha+\theta}\left[R \frac{1-e^{-\alpha T_{1}}}{1-e^{-\alpha T}}-y \frac{1-e^{-\alpha\left(T_{1}+T_{2}\right)}}{1-e^{-\alpha T}}\right] .
$$

The firm's annuity stream profit function is the sum of (12), (13), (14), minus the sum of (15), (16), (22), (23), (29), and (30): 


$$
\begin{aligned}
A S P & =(p-c) R-(g+r)(R-\beta y)+\frac{b(R-\beta y)}{\alpha}-\pi(1-\beta) y-\alpha s\left(1+\frac{e^{-\alpha\left(T-T_{4}\right)}}{1-e^{-\alpha T}}\right) \\
& +y\left[p-g \beta+\frac{d \theta+f}{\alpha+\theta}+\frac{b \beta}{\alpha}+\pi(1-\beta)\right] \frac{1-e^{-\alpha\left(T_{1}+T_{2}\right)}}{1-e^{-\alpha T}} \\
& -\left[(p-c-g-r) R+r \beta y+\frac{b R}{\alpha}\right] \frac{1-e^{-\alpha\left(T-T_{4}\right)}}{1-e^{-\alpha T}}-R\left[c+\frac{d \theta+f}{\alpha+\theta}\right] \frac{1-e^{-\alpha T_{1}}}{1-e^{-\alpha T}} .
\end{aligned}
$$

\subsection{Generalised demand}

The model so far has assumed that during a period of shortages the demand rate drops to $\beta y$, a fraction of the demand rate $y$ that holds in periods when there is positive stock. As such, it accounts for measuring what we call a direct financial effect of postponing income received (through backorders) as well as not realising a sale (through lost sales). Firms may in general, however, be also worried about what we call an indirect financial effect of shortages. This may arise when in the face of competition, alternative options would exist for customers to purchase the product. The exact nature of this effect will be contextdependent, and we can imagine many plausible ways of modelling it.

In this section we present one such an approach. It is based on the idea that a fraction of lost sales, say $\epsilon(0 \leq \epsilon \leq 1)$, would result in corresponding customers finding alternative options to purchase the product and not returning in the future. Those lost sales occur at a rate $(1-\beta) y$ during the period $T_{3}+T_{4}$ of an inventory cycle of length $T$. A simple approach to capturing this effect is to set the actual demand rate $y(\epsilon)$ as follows:

$$
y(\epsilon)=\left(1-\epsilon(1-\beta) \frac{T_{3}+T_{4}}{T}\right) y,
$$

where $y$ would be the demand rate when there are never shortages. Using (9) this simplifies to:

$$
y(\epsilon)=\left(1-\epsilon \frac{T_{3}}{T}\right) y
$$

This generalised demand function does not alter any of the previous calculations, and the profit function is still given by (25) when substituting $y(\epsilon)$ for $y$. 


\section{Equivalence Analysis}

Conditions are derived under which a cost minimising NPV model constructed from discounting the classic inventory parameters $h, h_{d}, h_{b}$ and $h_{l}$ will identify the same optimal inventory policy as a model based on maximising the NPV of the cash-flow functions derived in Section 3.3, i.e. for the case that $\gamma=1$.

\subsection{Opportunity holding cost models}

This section represents the approach found in models from the literature in which the opportunity cost

of investments in stock is incorporated in the unit holding cost $h$ (see also Section 1). We construct an NPV cost minimisation model for the activity as described in Section 2.1 from discounting costs of holding stock, backorders, lost sales, and deterioration, using parameters $h, h_{b}, h_{l}$, and $h_{d}$ :

$$
\begin{gathered}
H C=\left[\int_{0}^{T_{1}+T_{2}} h I(t) e^{-\alpha t} d t\right] \sum_{i=0}^{\infty} \alpha e^{-\alpha i T} \\
=\frac{h}{\alpha+\theta}\left[R \frac{1-e^{-\alpha T_{1}}}{1-e^{-\alpha T}}-y \frac{1-e^{-\alpha\left(T_{1}+T_{2}\right)}}{1-e^{-\alpha T}}\right] \\
B C=\left[\int_{T_{1}+T_{2}}^{T} h_{b} B(t) e^{-\alpha t} d t\right] \sum_{i=0}^{\infty} \alpha e^{-\alpha i T} \\
=\frac{h_{b} R}{\alpha}\left[\frac{1-e^{-\alpha\left(T-T_{4}\right)}}{1-e^{-\alpha T}}\right]-\frac{h_{b} \beta y}{\alpha}\left[\frac{1-e^{-\alpha\left(T_{1}+T_{2}\right)}}{1-e^{-\alpha T}}\right]-\frac{h_{b}}{\alpha}(R-\beta y), \\
L C=\left[\int_{T_{1}+T_{2}}^{T} h_{l}(1-\beta) y e^{-\alpha t} d t\right] \sum_{i=0}^{\infty} \alpha e^{-\alpha i T} \\
=h_{l} y(1-\beta)\left[1-\frac{1-e^{-\alpha\left(T_{1}+T_{2}\right)}}{\left.1-e^{-\alpha T}\right]}\right.
\end{gathered}
$$

and

$$
\begin{aligned}
D C & =\left[\int_{0}^{T_{1}+T_{2}} h_{d} \theta I(t) e^{-\alpha t} d t\right] \sum_{i=0}^{\infty} \alpha e^{-\alpha i T} \\
& =\frac{h_{d} \theta}{\alpha+\theta}\left[R \frac{1-e^{-\alpha T_{1}}}{1-e^{-\alpha T}}-y \frac{1-e^{-\alpha\left(T_{1}+T_{2}\right)}}{1-e^{-\alpha T}}\right] .
\end{aligned}
$$

The units of the classic parameters can be derived from these equations since each component calculated 
has to result in monetary units per unit of time. It follows from (33) that $h$ is a cost per unit of product and unit of time, from (34) that $h_{b}$ is a cost per unit of product and unit of time, from (35) that $h_{l}$ is a cost per unit of product and from (36) that $h_{d}$ is a cost per unit of product.

In addition, the annuity stream cost of set-ups is calculated in the same way as in the reference model and produces the result (15). The objective function is the sum of these components:

$$
\begin{aligned}
A S C & =h_{l}(1-\beta) y-\frac{h_{b}(R-\beta y)}{\alpha}+\alpha s\left(1+\frac{e^{-\alpha\left(T-T_{4}\right)}}{1-e^{-\alpha T}}\right) \\
& -y\left[\frac{h_{d} \theta+h}{\alpha+\theta}+\frac{h_{b} \beta}{\alpha}+h_{l}(1-\beta)\right] \frac{1-e^{-\alpha\left(T_{1}+T_{2}\right)}}{1-e^{-\alpha T}} \\
& +\frac{h_{b} R}{\alpha}\left[\frac{1-e^{-\alpha\left(T-T_{4}\right)}}{1-e^{-\alpha T}}\right]+\frac{R\left(h_{d} \theta+h\right)}{(\alpha+\theta)}\left[\frac{1-e^{-\alpha T_{1}}}{1-e^{-\alpha T}}\right] .
\end{aligned}
$$

Note that the model (37) captures the traditional inventory modelling approach, exemplified in its most simple form by the EOQ model (Harris, 1913), in which neither variable purchasing costs nor revenue streams are explicitly considered. We must hence for obvious reasons assume that demand $y$ is constant.

Theorem 1. Sufficient conditions for equivalence are:

$$
\begin{gathered}
h_{d} \theta+h=(d+c) \theta+\alpha c+f, \\
h_{b}=\alpha(p-c)-\alpha g-\alpha r\left(1-\frac{\beta y}{R}\right)+b, \\
h_{l}=(p-c)+r \frac{\beta}{1-\beta}\left(1-\frac{\beta y}{R}\right)+\pi .
\end{gathered}
$$

Proof. The proof is based on a variation to the algebraic derivation method developed in Grubbström (1996), or alternatively, to the difference approach in Grubbström (1998). Let $\Sigma$ be the sum of (31) and (37), i.e.: 


$$
\begin{aligned}
\Sigma & =(p-c) R-(g+r)(R-\beta y)-\frac{1}{\alpha}\left(h_{b}-b\right)(R-\beta y)+\left(h_{l}-\pi\right)(1-\beta) y \\
& +y\left[p-g \beta+\frac{\left(d-h_{d}\right) \theta+f-h}{\alpha+\theta}-\frac{\left(h_{b}-b\right) \beta}{\alpha}-\left(h_{l}-\pi\right)(1-\beta)\right] \frac{1-e^{-\alpha\left(T_{1}+T_{2}\right)}}{1-e^{-\alpha T}} \\
& -\left[(p-c-g-r) R+r \beta y-\frac{\left(h_{b}-b\right) R}{\alpha}\right] \frac{1-e^{-\alpha\left(T-T_{4}\right)}}{1-e^{-\alpha T}} \\
& -R\left[c+\frac{\left(d-h_{d}\right) \theta+f-h}{\alpha+\theta}\right] \frac{1-e^{-\alpha T_{1}}}{1-e^{-\alpha T}} .
\end{aligned}
$$

We now seek for the conditions under which $\Sigma$ is independent of the decision variables. It is observable that this is so when (38)-(40) hold: the coefficient in the last term of (41) is zero only when (38) holds, and the coefficient of the second to last term of (41) is zero only when (39) holds. Substitution of (39) into the coefficient of the third to last term of (41) then leads to (40) as the condition to make this coefficient zero. Hence, under these conditions it holds for sure that a policy that minimises $A S C$ will also be optimal for maximising $A S P$.

Some parts of the conditions (38)-(40) correspond to intuition reasonably well, as explained further below, but the exact function specifications for $h_{b}$ and $h_{s}$ in particular are very hard to establish if not having been explicitly derived. This underlines the value of NPVEA.

We now discuss the equivalence conditions in more detail. From (38) we see that there is a degree of freedom in how to set $h$ and $h_{d}$ in relation to each other. The most obvious solution is to take $h=\alpha c+f$ as this corresponds to the classic interpretation of this parameter, see Silver et al. (1998), from which it follows that $h_{d}=d+c$. The relevant disposal cost for every deteriorated item includes the initial purchasing $\operatorname{cost} c$. Note that in case that $d<0$ (a salvage value), its absolute value will typically be smaller than the cost price $c$, and hence $h_{d}>0$, as otherwise it would be economical to produce/purchase items for supplying a market of deteriorated items with a net marginal profit.

The first component of the unit backorder cost according to (39) is $\alpha(p-c)$, i.e. a capital cost of deferred marginal profits. Such a result has previously been found to hold in Grubbström (1998) in a stochastic setting. Our reference NPV model, by its inclusion of $g$ and $r$, shows that the functional form of $h_{b}$ can be more complicated. Any non-zero deposit $g$ reduces the capital loss on backordered sales and this is quite intuitive. Any non-zero $r$ for backordered items reduces the capital cost of $h_{b}($ since $1-\beta y / R>0)$. This 
is perhaps less intuitive at first sight. The unit backorder cost, however, only measures the opportunity cost of $r$. Because it is a deferred cost, it must indeed be that this opportunity cost is negative and hence that an increase in $r$ reduces $h_{b}$. The reason why the overall effect of an increasing $r$ will negatively affect a firm's profits is because it also appears in the constant cost terms in (31). Finally, there is a cost $b$ that is similar in interpretation as the $f$ in $h$ : an out-of-pocket backorder cost the moment the backordered demand occurs. Since it must be that $p-g-r-c>0$ for backordering to make economical sense, we find $h_{b}>0$.

The lost sales cost $h_{l}$ as given by (40) demonstrates the importance to account for the loss in profits through $p-c$, but shows in addition the somewhat peculiar impact from the cost $r$ that is experienced for items that are sold with backordering. There is no easy interpretation of this result, as discussed in Section 4.3: it is a mathematical construct needed to 'correct' the approach of discounting classic parameters as to ensure that it will optimise the NPV of the profit function of the firm. Similar counter-intuitive results also occur in the context of remanufacturing, see e.g. Çorbacioğlu and van der Laan (2007).

Note that, while sufficient, we have not proven the mathematical necessity nor uniqueness of the derived conditions (38)-(40) for equivalence to hold for only optimal solutions. Given the reasonably intuitive explanations for most of the components in the identified equivalence conditions, we did not investigate the existence of other potential (and then probably exotic) solutions under which equivalence may hold in the strict sense. The equivalence conditions derived do preserve the relative comparison of any solution to an optimum, and are hence arguably of practical relevance. Numerical experiments in which the derived equivalence conditions are used confirm the accuracy of these equivalence conditions, see also Section 5.3.

\subsection{Holding cost models excluding opportunity costs}

In this section a cost minimising NPV model is derived following the stream of models in the literature (see also Section 1) that model the purchasing/production costs explicitly. Exactly the same calculations (33)-(36) are used as in Section 4.1 for the costs related to parameters $h, h_{b}, h_{l}, h_{d}$, and (15) related to $s$. In addition, the annuity stream production cost is explicitly modelled as in (16). We again assume in our 
model that $y$ is constant, and that revenue streams are not explicitly modelled.

Theorem 2. Sufficient conditions for equivalence are:

$$
\begin{gathered}
h_{d} \theta+h=d \theta+f, \\
h_{b}=\alpha p-\alpha g-\alpha r\left(1-\frac{\beta y}{R}\right)+b, \\
h_{l}=p+r \frac{\beta}{1-\beta}\left(1-\frac{\beta y}{R}\right)+\pi .
\end{gathered}
$$

Proof. Details are omitted, as it follows the approach as before based on:

$$
\begin{aligned}
\Sigma & =p R-(g+r)(R-\beta y)-\frac{1}{\alpha}\left(h_{b}-b\right)(R-\beta y)+\left(h_{l}-\pi\right)(1-\beta) y \\
& +y\left[p-g \beta+\frac{\left(d-h_{d}\right) \theta+f-h}{\alpha+\theta}-\frac{\left(h_{b}-b\right) \beta}{\alpha}-\left(h_{l}-\pi\right)(1-\beta)\right] \frac{1-e^{-\alpha\left(T_{1}+T_{2}\right)}}{1-e^{-\alpha T}} \\
& -\left[(p-g-r) R+r \beta y-\frac{\left(h_{b}-b\right) R}{\alpha}\right] \frac{1-e^{-\alpha\left(T-T_{4}\right)}}{1-e^{-\alpha T}} \\
& -R\left[\frac{\left(d-h_{d}\right) \theta+f-h}{\alpha+\theta}\right] \frac{1-e^{-\alpha T_{1}}}{1-e^{-\alpha T}} .
\end{aligned}
$$

End of proof. $\diamond$

Interpretations can be derived as previously in Section 4.1. From (42) we take $h=f$ and $h_{d}=d$. The opportunity cost of capital is no longer to be included into the unit holding cost, and the unit deterioration cost only accounts for the disposal cost/salvage value (hence, $h_{d}<0$ is now possible). As the impact of purchasing/production is explicitly modelled its impact should not only be excluded from $h$ but also from $h_{b}$ and $h_{l}$. 


\subsection{The non-triviality of the equivalence results}

It should be emphasised that the equivalence results obtained are non-trivial. Indeed, the general expression for discounting classic cost parameters as in (33) is:

$$
\int_{0}^{\infty} h I(t)^{+} \alpha e^{-\alpha t} d t
$$

This is not conform to the standard definition of calculating an annuity stream value, which requires that it should be based on: (A) only real cash-flows, and (B) the exact timing of these real cash-flows. However, discounting $h$ as above satisfies neither of these conditions. Indeed, we have just found that, for equivalence to hold, it must be that $h=\alpha c+f$, and hence by virtue of containing a capital cost $\alpha c$ this does not satisfy condition (A). Furthermore, (46) does also not respect condition (B): for $R \rightarrow \infty$, for example, the purchasing/production cost occurs at the start of a cycle and not continuously throughout the cycle. It is therefore surprising that these two wrong assumptions about the cash-flow structure are able to neutralise each other so that the end result can still be made compatible with the standard NPV criterion. Similarly, we should be surprised to have found that a similar operation on e.g. the unit backorder cost, as in (34) still works, despite the fact that the equivalence conditions again state that $h_{b}$ should include capital costs in its formula and hence also violates both conditions (A) and (B). We note that the discounting of classic parameters is not restricted to the literature on deterioration but occurs in other fields as well.

\section{$5 \quad$ Numerical examples}

\subsection{Financial impact of shortages under constant demand}

This section illustrates the use of the NPV cash-flow model developed in Section 3 to examine the financial implications of shortages when $y$ is constant. A factorial design is set-up testing for five factors, of which $p$ is set at four levels and the other four factors are set at two levels each: $p$ at $3 c, 2 c, 1.5 c$ and $1.3 c ; \beta$ at 0.9 and $0.5 ; \theta$ at 0 (i.e. no deterioration) and $0.05 ; g$ at 0 and $0.1 p$; and $r$ at 0 and $0.1 p$, respectively. This produces 64 experiments, where in each case the other parameters are set at the following values: 
$\alpha=0.08 ; y=100 ; s=80 ; f=2.3 ; b=1 ; c=5 ; \pi=0 ; d=0 ; \gamma=1$, and $R=160$.

We consider two models: the first is the reference model of Section 3 in which shortages are allowed, the second is the same model but in which shortages are 'ex-ante' prohibited (i.e. $T_{3}=T_{4} \equiv 0$ per definition). As the independent parameters are $T_{2}$ and $T_{3}$ only, see Section 3.2, we have set up a simple exhaustive search routine in two nested loops, whereby values for these parameters are incremented across a range. While not expecting this to produce the most efficient running times, all scenarios are solved fairly quick even with small stepsizes.

For each of the 64 scenarios, we calculate $\delta=100\left(A S P_{N S}^{*}-A S P^{*}\right) / A S P^{*}$, the percentage optimality gap between the profits of reference model's optimal solution $A S P^{*}$ and the profits $A S P_{N S}^{*}$ of the noshortages-allowed version's optimal solution. The more negative the value of $\delta$, the larger the financial benefit of planning for shortages. A summary of main results is given in Table 1. If the profit margin is high $(p=3 c)$, the reference model indicates that in 12 out of the 16 experiments it is financially best not to plan for shortages, and in the other 4 cases the benefit of doing so is less than $1 \%$ (i.e. $\delta \geq-1.00$ ).

For prices set at the two intermediate levels ( $p$ at $2 c$ and $1.5 c$ ), the most important factor is the fraction of shortages that leads to backorders $(\beta)$. For $\beta=0.5$, the financial benefit of planning for shortages is zero in 12 out of the 16 scenarios, and in the remaining four cases (for $p$ at $1.5 c$ ) the benefit remains significantly smaller than $1 \%$. At modest lost sales ratios $(\beta=0.9)$, however, the profit margin is an important consideration: a modest benefit from shortages occurs for prices set at $p=2 c$, in particular when no reduction on sales price has to be given $(r=0.0)$, however this benefit grows to larger values and up to about $25 \%$ for $r=0.0$ when the price is set at $p=1.5 c$. The parameters with no significant impact are $g$ and $\theta$. In all scenarios tested at these price settings, the benefit reduces when customers place deposits $(g=0.1)$, but the impact on $\delta$ value changes always remains smaller than $0.5 \%$. The impact of $\theta$ is somewhat larger but still quite small: if the product deteriorates it is somewhat more beneficial to plan for shortages but in all scenarios this impact remains below $2.5 \%$.

If the profit margin is modest $(p=1.3 c)$, the benefit of planning for shortages ranges between about $24 \%$ to $78 \%$ and other factors come into play. The second most important factor in those cases is $\beta$ since the financial benefit of shortages will increase the less shortages result in lost sales. More specifically, when 
Table 1: Summary of experiments of Section 5.1

\begin{tabular}{llllll}
\hline$p$ & $\beta$ & $r$ & $\theta$ & $g$ & Average $\delta(\%)$ \\
\hline $3 c$ & - & - & - & - & -0.11 \\
$2 c$ & 0.5 & - & - & - & -0.00 \\
$2 c$ & 0.9 & 0.1 & - & - & -2.07 \\
$2 c$ & 0.9 & 0.0 & - & - & -6.08 \\
$1.5 c$ & 0.5 & - & - & - & -0.16 \\
$1.5 c$ & 0.9 & 0.1 & - & - & -16.88 \\
$1.5 c$ & 0.9 & 0.0 & - & - & -25.34 \\
$1.3 c$ & 0.5 & 0.1 & - & - & -30.69 \\
$1.3 c$ & 0.5 & 0.0 & - & - & -49.51 \\
$1.3 c$ & 0.9 & - & 0.0 & - & -65.08 \\
$1.3 c$ & 0.9 & - & 0.05 & - & -73.91 \\
\hline
\end{tabular}

shortages lead to high lost sales $(\beta=0.5)$, it then largely depends on a third factor $r$. If customers receive $10 \%$ off the price when experiencing a backorder, the average financial benefit of shortages is $30.69 \%$, but this increases to an average of $49.51 \%$ when no financial compensation has to be given $(r=0.0)$. Whether or not the product deteriorates is still quite significant: if the product deteriorates $(\theta=0.05 \%)$ it will add on average about $12 \%$ to the gap. If shortages lead to low lost sales $(\beta=0.9)$, the most important factor is not $r$ but whether or not the product deteriorates. If products do not deteriorate, planning for shortages produces on average a financial benefit of $65.08 \%$, but if products deteriorate $(\theta=0.05)$ this increases to 73.91\%. The impact of $r$ is now on average about 7\%, and is therefore still almost as important as the product's deterioration characteristic.

Note that in the experiments above there is no cost for the disposal of deteriorated items $(d=0)$. Naturally, for larger values of $d$ the financial benefit of planning for shortages when the product deteriorates will increase. We therefore repeated the above experiments for $d=0.2 c$, but found that its impact on affecting $\delta$ values remains below $1 \%$.

In conclusion, the experiment illustrates how an NPV model based on cash-flow functions can be used to examine the potential financial benefits of planning for shortages. For the parameter settings investigated, it appears that if products are sold at large profit margins of $3 c$, then planning for shortages is not meaningful under all circumstances. For lower profit margins, the fraction of shortages leading to backorders $(\beta)$ is the most important factor. The benefit of planning for shortages becomes significant for 
prices around $1.5 c$ to $1.3 c$, with the benefit increasing the lower the price margin, the higher $\beta$, and the smaller the discount customers will receive on the backordered item. If products are sold at a lower markup of $30 \%$, and if there is evidence that most customers will accept backorders, planning for shortages carries a significant financial benefit which increases the more the product deteriorates. The area in which it is most difficult to predict the financial benefit of shortages is when products are sold at modest profit margins and when shortages result in a reasonable fraction of lost sales. It will then also be significantly influenced by any discount on the backordered item. In all cases, whether or not the customers pay a deposit has very little financial consequences (the main effect of $g$ is less than $1 \%$ ). However in practise it may be a worthwhile mechanism as a means to secure the backorder so that customers will not change their mind and become a lost sale. The impact of the unit cost to remove deteriorated items remains small.

\subsection{Financial impact of shortages for generalised demand}

The assumption of constant demand is relaxed and replaced by the demand function presented in Section 3.4. We use the set-up of Section 5.1, but since the impact of $g$ appeared to be very small, we drop this factor in the analysis and keep it constant at $g=0.0$, and replace it with the factor $\epsilon$, set at three levels: 0.20, 0.1 and 0.01 , respectively. We restrict sales price to its two extremes, $p=3 c$ and $p=1.3 c$. This leads in total to 48 experiments.

The results are summarised in Table 2. For $p=3 c$, the reference model indicates that in 22 out of the 24 experiments it is financially best not to plan for shortages, and in the other 2 cases the benefit of doing so is less than $0.5 \%$. Comparing with the results in Table 1 , we can infer that the benefit of planning for shortages becomes negligible for $p=2 c$ and $\epsilon \geq 0.10$. For $p=1.3 c$, the benefit of planning for shortages ranges between about $1.2 \%$ to $74 \%$. Results support the intuition that this demand sensitivity reduces the benefit of planning for shortages the larger the fraction of lost sales, i.e., the smaller the value of $\beta$. When shortages lead to high lost sales $(\beta=0.5)$, the impact of a higher sensitivity of demand to lost sales $(\epsilon \geq 0.10)$ quite significantly interacts with $r$, i.e., whether or not the customers receive $10 \%$ off the price. When shortages lead to low lost sales $(\beta=0.9)$, the impact of $\epsilon$ in the tested range is approximately 
of equal magnitude to the impact of $\theta$ and $r$, but in any case the financial advantages for planning for shortages remains high.

Table 2: Summary of experiments of Section 5.2.

\begin{tabular}{llllll}
\hline$p$ & $\beta$ & $r$ & $\theta$ & $\epsilon$ & Average $\delta(\%)$ \\
\hline $3 c$ & - & - & - & - & -0.03 \\
$1.3 c$ & 0.5 & 0.1 & - & 0.20 & -4.78 \\
$1.3 c$ & 0.5 & 0.1 & - & 0.10 & -18.52 \\
$1.3 c$ & 0.5 & 0.1 & - & 0.01 & -29.29 \\
$1.3 c$ & 0.5 & 0.0 & - & 0.20 & -31.84 \\
$1.3 c$ & 0.5 & 0.0 & - & 0.10 & -41.69 \\
$1.3 c$ & 0.5 & 0.0 & - & 0.01 & -48.53 \\
$1.3 c$ & 0.9 & - & 0.0 & 0.20 & -58.43 \\
$1.3 c$ & 0.9 & - & 0.0 & 0.10 & -61.91 \\
$1.3 c$ & 0.9 & - & 0.0 & 0.01 & -64.55 \\
$1.3 c$ & 0.9 & - & 0.05 & 0.20 & -68.49 \\
$1.3 c$ & 0.9 & - & 0.05 & 0.10 & -72.16 \\
$1.3 c$ & 0.9 & - & 0.05 & 0.01 & -73.48 \\
\hline
\end{tabular}

To conclude, the experiment illustrates the importance of accounting for the indirect financial effect of shortages when the profit margins are modest and when a significant fraction of shortages lead to lost sales. In those circumstances the interaction effect with the discount level offered to backorders is significant. Planning for shortages has potential in the presence of lost sales-level demand sensitivity for low profit margins and high backorder rates, but its impact depends much on the interaction with both the discount level and the product's deterioration characteristics.

\subsection{Using a model based on unit cost parameters $h, h_{b}, h_{l}$ and $h_{d}$}

All previous experiments were conducted with the cash-flow based profit maximising NPV model developed in Section 3. For negative exponential deterioration $(\gamma=1)$, we can instead use one of the two cost minimising NPV models based on discounting the classic inventory cost parameters presented in Section 4. We have hence repeated the experiments for a constant demand while setting the four cost parameters $h, h_{b}, h_{l}$, and $h_{d}$ to values given by Theorems 1 and 2 , respectively, and found that the optimal inventory policies derived as such are indeed exactly equal to those found from the cash-flow NPV model. This 
provides a numerical confirmation that the equivalence conditions are accurate.

When calculating $\delta$ values based on the cost functions of these models, it was found that the relative cost savings achieved do not at all correspond to the profit savings found from the cash-flow based NPV reference model and which are reported in the above sections. If not interpreted carefully, it will lead to the wrong insights. For example, the cost minimising model indicates that logistics costs reduce by increasing $r$. This does not mean it is beneficial to increase $r$ at all, since overall profits would go down, as discussed in Section 4.1. The reasons for this are that important terms that affect profits are still not incorporated into the cost minimising models. This is also clear from e.g. the difference equations (41) and (45), of which the first four terms are not represented in the cost minimising models. Reliable recommendations are hence difficult to achieve with cost minimisation models based on the unit cost parameters, even if equivalence conditions are known.

If the equivalence conditions are not known, then the power of an NPV model discounting these unit cost parameters is very poor. In particular for the system studied in this paper, such models will not correctly identify how the optimal policy changes for different values of $R, \beta$, or $y$, if $h_{b}$ and $h_{l}$ are treated as exogeneous parameters and are kept constant. This is unfortunately the approach adopted in the current literature. We prove in this paper that obtaining the correct insight into the sensitivity to $R, \beta$ and $y$ requires knowledge about their relationship to $h_{b}$ and $h_{l}$. We have shown how this can be done via NPVEA. Since this means constructing also a cash-flow based NPV model, however, one can in effect use this model rather than a model based on $h_{b}$ and $h_{l}$.

We also conducted a series of experiments for general Weibull-based deterioration. The aim was to test whether the equivalence conditions derived in Section 4 for the special case that $\gamma=1$ would reasonably work for more general deterioration patterns. In particular, we tested for the case that $\gamma=1.5$ and compared the profits and cycle time values obtained from the cash-flow NPV model with that obtained from a cost minimising model in which we still use Theorems 1 and 2 to set the cost parameters $h, h_{b}$, $h_{l}$, and $h_{d}$. We found that these equivalence conditions now do no longer produce an accurate match and that the numerical values of in particular $h$ and $h_{b}$ are to be set differently in order to minimise the error with the cash-flow based NPV model. This tells us that these classic parameters are now very likely 
also a function of the endogeneous parameters that govern the deterioration. As equivalence conditions for $\gamma \neq 1$ are much harder to establish, we did not attempt to find these relationships. These findings, however, provide further indirect evidence in support of the view that if classic inventory parameters are discounted in NPV models, one cannot be sure that these parameters are in general exogeneous to the model. Adopting cash-flow based NPV models altogether in this sense offers a route towards a more reliable inventory theory.

\section{Conclusions}

How can deterministic inventory models provide quantitative insights into the economics of system design choices, such as whether a firm would derive financial benefits from planning for shortages, or what the impact would be from changing its production rate, or of the discount offered on backorders? This paper

has reinforced that cash-flow based Net Present Value (NPV) models are more reliable for deriving such insights than models based on the classic inventory parameters. We have done this by showing that, in the system studied in this paper, $h_{b}$ and $h_{l}$ ought to receive specific values as specified through non-trivial functions of other parameters in the model in order to find the optimal inventory policy. Cash-flow based NPV models have the advantage of not having to rely on these classic parameters.

A cash-flow based NPV model is developed for the case of a deteriorating item produced at a finite production rate. Regarding the value of planning for shortages, results show that the value of planning for shortages is most significant when the profit margin is small and when the fraction of backorders remains high. In those situations, it is also important to know the level of the discount offered on backorders, the item's deterioration characteristics, and the degree to which lost sales leads to lost demand in general. For high profit margins of twice the unit purchase or production cost of a product, planning for shortages does not offer significant financial benefits. Whether or not customers pay a $10 \%$ deposit for backordered items, and whether or not there would be a $20 \%$ disposal cost for deteriorated items, appear insignificant. These findings may not generalise to other firm-specific situations, and we recommend the use of cash-flow based NPV models as developed in this paper. 
Further research in this area should concentrate on increasing our understanding of how backorders for items with low-profit margins influence future demand. Despite the potential value of planning for backorders identified in the model in such situations, it is intuively clear that if stockouts are not appreciated by customers, models need further refinement as to take this aspect into account but without resorting to artificial tweaking of parameters ${ }^{3}$.

The model developed was in addition used to gain insight into the nature of classic parameters $h_{b}$ and $h_{l}$. This can be done since the model is based on cash-flow functions and does not rely on these classic parameters. This approach captures the essence of NPVEA as described in Beullens and Janssens (2014). While the equivalence conditions derived in Section 4 show that it is possible to use an NPV model based on $h, h_{b}$ and $h_{l}$ to calculate an inventory policy that is also optimal for the NPV cash-based model, these equivalence relationships also indicate that the classic inventory parameters are not exogenously determinable. As $h_{b}$ and $h_{l}$ depend on both financial and non-financial system parameters, no reliable insights from sensitivity analysis would result from models using these parameters if these equivalence conditions are not known.

The derived functional specifications in Section 4 for $h, h_{b}, h_{d}$ and $h_{l}$ should not be taken to hold firmly in all models which use them. In deriving these results, NPVEA has to rely on both a cash-based reference NPV model, and another model which uses the classic parameters. If one of these two models change, then so may the equivalence conditions needed. What this also means is that different models which use the classic parameters $h, h_{b}, h_{d}$ and $h_{l}$ may in fact also need a different interpretation of these parameters. This complicates the proper comparison of classic models as well as makes it very hard to derive any insight into these parameters. The findings in this paper clearly support the observation made in Winston (1994) about the difficulty of quantifying these parameters in practise, as discussed in Section 1, but shows in addition that NPVEA can also explain why this is so difficult. The difference between Theorems 1 and 2 , as well as the experiments for different deterioration patterns in Section 5.3 illustrate this point. Further research is to be conducted to see how the equivalence conditions look like when comparing the reference model in this paper with average cost models.

\footnotetext{
${ }^{3}$ For example, while increasing $h_{b}$ is a easy 'fix' to reduce backorders in the 'optimal' policy, this approach offers no satisfactory solution as the policy is then only optimal in a mathematical sense and the model looses its explanatory power.
} 
The findings of this paper support a general message found in the NPVEA literature which, at this point, still seems somewhat undervalued. The paper contributes to an increasing body of evidence that inventory theory suffers from its reliance on traditional parameters $h, h_{b}$, and $h_{l}$ to capture economic tradeoffs, a concept that is central to any theory of inventories. In particular this paper shows that the theory suffers from the (implicit) assumption that these classic parameters are independent and exogeneous to

the model, because they may not be. Another general contribution of the paper is specifically adressing the body of NPV literature based on discounting these classic parameters. This approach is not an improvement over average cost models as it still relies on these difficult to interpret parameters. This method further violates the standard assumptions of NPV theory, as discussed in Section 4.3. The cashflow NPV approach does not suffer from these drawbacks. It has been available to us for at least half a century, and shown its relative value in comparison to using classic inventory parameters as early as Grubbström (1980).

\section{References}

Aggarwal, S.P., C.K. Jaggi. 1995. Ordering policies of deteriorating items under permissible delay in payments. The Journal of the Operational Research Society 46(5) 658-662.

Bakker, M., J. Riezebos, R.H. Teunter. 2012. Review of inventory systems with deterioration since 2001. European Journal of Operational Research 221 275-284.

Balkhi, Z.T. 2011. Optimal economic ordering policy with deteriorating items under different supplier trade credits for finite horizon case. International Journal of Production Economics 133 216-223.

Beullens, P. 2014. Revisiting foundations in lot sizing - Connections between Harris, Crowther, Monahan, and Clark. International Journal of Production Economics 155 68-81.

Beullens, P., G.K. Janssens. 2011. Holding costs under push or pull conditions - The impact of the Anchor Point. European Journal of Operational Research 215 115-125. 
Beullens, P., G.K. Janssens. 2014. Adapting inventory models for handling various payment structures using Net Present Value Equivalence Analysis. International Journal of Production Economics 157 190-200.

Boyaci, T., G. Gallego. 2002. Coordinating pricing and inventory replenishment policies for one wholesaler and one or more geographically dispersed retailers. International Journal of Production Economics $\mathbf{7 7}$ $95-111$.

Çorbacioğlu, U., E.A. van der Laan. 2007. Setting the holding cost rates in a two-product system with remanufacturing. International Journal of Production Economics 109 185-194.

Chang, C.T., L.Y. Ouyang, J.T. Teng, M.C. Cheng. 2010. Optimal ordering policies for deteriorating items using a discounted cash-flow analysis when a trade credit is linked to order quantity. Computers Ef Industrial Engineering 59 770-777.

Chern, M.S., H.L. Yang, J.T. Teng, S. Papachristos. 2008. Partial backlogging inventory lot-size models for deteriorating items with fluctuating demand under inflation. European Journal of Operational Research $191127-141$.

Chung, K.J., J.J. Liao. 2006. The optimal ordering policy in a DCF analysis for deteriorating items when trade credit depends on the order quantity. International Journal of Production Economics 100 $116-130$.

Dye, C.Y., L.Y. Ouyang, T.P. Hsieh. 2007. Inventory and pricing strategies for deteriorating items with shortages: A discounted cash flow approach. Computers \& Industrial Engineering 52 29-40.

Goyal, S.K., B.C. Giri. 2001. Recent trends in modeling of deteriorating inventory. European Journal of Operational Research $1341-16$.

Grubbström, R.W. 1967. On the application of the Laplace transform to certain economic problems. Management Science 13(7) 558-567. 
Grubbström, R.W. 1980. A principle for determining the correct capital costs of work-in-progress and inventory. International Journal of Production Research 18(2) 259-271.

Grubbström, R.W. 1996. Material requirements planning and manufacturing resource planning. M. Warner, ed., International Encyclopedia of Business and Management. Routeledge, London, 34003420.

Grubbström, R.W. 1998. A net present value approach to safety stocks in planned production. International Journal of Production Economics 56-57 213-229.

Harris, F.W. 1913. How many parts to make at once. Factory, The Magazine of Management 10(2) $135-136,152$.

Hou, K.L. 2006. An inventory model for deteriorating items with stock-dependent consumption rate and shortages under inflation and time discounting. European Journal of Operational Research 168 463-474.

Hou, K.L., L.C. Lin. 2011. A supply chain model for deteriorating items with time discounting under trade credit and quantity discounts. African Journal of Business Management 5(25) 10243-10251.

Hsieh, T.P., C.Y. Dye. 2010. Pricing and lot-sizing policies for deteriorating items with partial backlogging under inflation. Expert Systems with Applications 37 7234-7242.

Hsieh, T.P., C.Y. Dye, L.Y. Ouyang. 2008. Determining optimal lot size for a two-warehouse system with deterioration and shortages using net present value. European Journal of Operational Research 191 $182-192$.

Jaggi, C.K., K.K. Aggarwal, S.K. Goel. 2006. Optimal order policy for deteriorating items with inflation induced demand. International Journal of Production Economics 103 707-714.

Jaggi, C.K., S.P. Aggarwal. 1994. Credit financing in economic ordering policies of deteriorating items. International Journal of Production Economics 34 151-155. 
Law, S.T., H.M. Wee. 2006. An integrated production-inventory model for ameliorating and deteriorating items taking account of time discounting. Mathematical and Computer Modelling 43 673-685.

Li, R., H. Lan, J.R. Mawhinney. 2010. A review on deteriorating inventory study. Service Science and Management 3 117-129.

Liao, H.C., C.H. Tsai, C.T. Su. 2000. An inventory model with deteriorating items under inflation when a delay in payment is permissible. International Journal of Production Economics 63 207-214.

Liao, J.J., K.N. Huang. 2010. An inventory model for deteriorating items with two levels of trade credit taking account of time discounting. Acta Applicandae Mathematicae 110 313-326.

Lo, S.T., H.M. Wee, W.C. Huang. 2007. An integrated production-inventory model with imperfect production processes and Weibull distribution deterioration under inflation. International Journal of Production Economics 106 248-260.

Moon, I., B.C. Giri, B. Ko. 2005. Economic order quantity models for ameliorating/deteriorating items under inflation and time discounting. European Journal of Operational Research 162 773-785.

Nahmias, S. 1982. Perishable inventory theory: A review. Operations Research 30(4) 680-708.

Raafat, F. 1991. Survey of literature on continuously deteriorating inventory models. Journal of the Operational Research Society 42(1) 27-37.

Sarker, B. R., A. M. M. Jamal, S. Wang. 2000. Supply chain models for perishable products under inflation and permissible delay in payment. Computers $\&$ Operations Research 27 59-75.

Silver, E.A., D.F. Pyke, R. Peterson. 1998. Inventory Management and Production Planning and Scheduling, 3th. ed.. John-Wiley and Sons, New York.

Singh, S.R., N. Kumar, R. Kumari. 2009. Two-warehouse inventory model for deteriorating items with shortages under inflation and time-value of money. International Journal of Computational and Applied Mathematics 4(1) 83-94. 
Singha, S.R., V. Gupta, P. Gupta. 2013. Three stage supply chain model with two warehouse, imperfect production, variable demand rate and inflation. International Journal of Industrial Engineering Computations 4 81-92.

Pentico, D.W., M.J. Drake. 2009. The deterministic EOQ with partial backordering: A new approach. European Journal of Operational Research 194 102-113.

Taleizadeh, A.A., M. Nematollahi. 2014. An inventory control problem for deteriorating items with backordering and financial considerations. Applied Mathematical Modelling 38 93-109.

Teunter, R., E. van der Laan. 2002. On the non-optimality of the average cost approach for inventory models with remanufacturing. International Journal of Production Economics 79 67-73.

Wee, H.M., S.T. Law. 2001. Replenishment and pricing policy for deteriorating items taking into account the time-value of money. International Journal of Production Economics 71 213-220.

Wee, H.M., J.C.P. Yu, S.T. Law. 2005. Two-warehouse inventory model with partial backordering and Weibull distribution deterioration under inflation. Journal of the Chinese Institute of Industrial Engineers 22(6) 451-462.

Winston, W.L. 1994. Operations Research: Applications and Algorithms. 3rd ed. Duxbury Press, Belmont,California.

Yang, H.L. 2004. Two-warehouse inventory models for deteriorating items with shortages under inflation. European Journal of Operational Research 157 344-356.

Yang, H.L. 2006. Two-warehouse partial backlogging inventory models for deteriorating items under inflation. International Journal of Production Economics 103 362-370.

Yang, H.L. 2012. Two-warehouse partial backlogging inventory models with three-parameter Weibull distribution deterioration under inflation. International Journal of Production Economics 138 107-116. 
Yang, H.L., J.T. Teng, M.S. Chern. 2010. An inventory model under inflation for deteriorating items with stock-dependent consumption rate and partial backlogging shortages. International Journal of Production Economics 123 8-19.

Yu, J.C.P. 2013. A collaborative strategy for deteriorating inventory system with imperfect items and supplier credits. International Journal of Production Economics 143 403-409.

Zipkin, P.H. 2000. Foundations in Inventory Management. McGraw-Hill, New York.

\section{A An approximation for inventory holding cost}

One may use the following Maclaurin expansion approximations for (2) and (4) as in Wee and Law (2001):

$$
\begin{gathered}
I(t)=(R-y) e^{-\theta t^{\gamma}}\left(t+\frac{\theta t^{\gamma+1}}{\gamma+1}\right), \quad 0 \leq t \leq T_{1}, \\
I(t)=y e^{-\theta t^{\gamma}}\left(T_{1}+T_{2}-t+\frac{\theta}{\gamma+1}\left(\left(T_{1}+T_{2}\right)^{\gamma+1}-t^{\gamma+1}\right)\right), \quad T_{1} \leq t \leq T_{1}+T_{2} .
\end{gathered}
$$

If we use the approximations (47) and (48), then $T_{1}=g\left(T_{2}\right)$ is implicit in:

$$
\left(1-\frac{y}{R}\right) T_{1}+\frac{\theta T_{1}^{\gamma+1}}{\gamma+1}=\frac{y}{R}\left(T_{2}+\frac{\theta}{\gamma+1}\left(T_{1}+T_{2}\right)^{\gamma+1}\right)
$$

and the values for $H C_{1}$ and $H C_{2}$ are given by:

$$
\begin{aligned}
H C_{1} & =f \int_{0}^{T_{1}} I(t) e^{-\alpha t} d t \\
& \approx f(R-y)\left(\frac{T_{1}^{2}}{2 T}-\frac{\alpha T_{1}^{3}}{3 T}-\frac{\theta \gamma T_{1}^{\gamma+2}}{(\gamma+1)(\gamma+2) T}+\frac{\alpha T_{1}^{2}}{4}\right)
\end{aligned}
$$


and

$$
\begin{aligned}
H C_{2}= & f \int_{T_{1}}^{T_{1}+T_{2}} I(t) e^{-\alpha t} d t \\
\approx & f y\left[\frac{T_{2}\left(T_{1}+T_{2}\right)}{T}+\frac{\alpha T_{2}}{2}\left(T_{1}+T_{2}\right)-\frac{1}{2}\left(\frac{1}{T}+\frac{\alpha}{2}\right)\left(\left(T_{1}+T_{2}\right)^{2}-T_{1}^{2}\right)\right. \\
& +\frac{\theta T_{2}}{T(\gamma+1)}\left(T_{1}+T_{2}\right)^{\gamma+1}-\frac{\theta}{T(\gamma+1)(\gamma+2)}\left(\left(T_{1}+T_{2}\right)^{\gamma+2}-T_{1}^{\gamma+2}\right) \\
& -\frac{\alpha\left(T_{1}+T_{2}\right)}{2 T}\left(\left(T_{1}+T_{2}\right)^{2}-T_{1}^{2}\right)+\frac{\alpha}{3 T}\left(\left(T_{1}+T_{2}\right)^{3}-T_{1}^{3}\right) \\
& \left.-\frac{\theta\left(T_{1}+T_{2}\right)}{T(\gamma+1)}\left(\left(T_{1}+T_{2}\right)^{\gamma+1}-T_{1}^{\gamma+1}\right)+\frac{\theta}{T(\gamma+2)}\left(\left(T_{1}+T_{2}\right)^{\gamma+2}-T_{1}^{\gamma+2}\right)\right] .
\end{aligned}
$$

